\title{
Nutritional demands in acute and chronic illness
}

\author{
Rosemary A. Richardson* and H. Isobel M. Davidson \\ Dietetics, Nutrition and Biological Sciences, Queen Margaret University College, Clerwood Terrace, \\ Edinburgh EH12 8TS, UK
}

\begin{abstract}
Common to both acute and chronic disease are disturbances in energy homeostasis, which are evidenced by quantitative and qualitative changes in dietary intake and increased energy expenditure. Negative energy balance results in loss of fat and lean tissue. The management of patients with metabolically-active disease appears to be simple; it would involve the provision of sufficient energy to promote tissue accretion. However, two fundamental issues serve to prevent nutritional demands in disease being met. The determination of appropriate energy requirements relies on predictive formulae. While equations have been developed for critically-ill populations, accurate energy prescribing in the acute setting is uncommon. Only 25-32\% of the patients have energy intakes within $10 \%$ of their requirements. Clearly, the variation in energy expenditure has led to difficulties in accurately defining the energy needs of the individual. Second, the acute inflammatory response initiated by the host can have profound effects on ingestive behaviour, but this area is poorly understood by practising clinicians. For example, nutritional targets have been set for specific disease states, i.e. pancreatitis $105-147 \mathrm{~kJ}(25-35 \mathrm{kcal}) / \mathrm{kg}$; chronic liver disease $147-168 \mathrm{~kJ}(35-40 \mathrm{kcal}) / \mathrm{kg}$, but given the alterations in gut physiology that accompany the acute-phase response, targets are unlikely to be met. In cancer cachexia attenuation of the inflammatory response using eicosapentaenoic acid results in improved nutritional intake and status. This strategy poses an attractive proposition in the quest to define nutritional support as a clinically-effective treatment modality in other disorders.
\end{abstract}

Acute and chronic disease: Energy homeostasis: Inflammatory response: Nutritional support

In both acute and chronic disease there are disturbances in energy homeostasis in which the consequences of the inflammatory response may affect both sides of the energy balance equation. For example, following trauma or major surgery, tissue injury and the resultant systemic inflammation may induce hypermetabolism. This outcome is also apparent in pathologies such as acute and chronic pancreatitis, inflammatory bowel disease and cancer. In such cases energy demands may also be altered, as evidenced by quantitative and qualitative changes (Davidson et al. 1999) in dietary intake in addition to increased energy expenditure (Schols, 2001).

It has been suggested that the presence of pathologies that induce a metabolic response contributes not only to diseaserelated undernutrition but may compound co-morbidity driven by poor immune function. The resolution of these anomalies of energy metabolism would seem simplistic, in that the provision of nutritional support (oral enteral or parenteral) sufficient to meet requirements would serve to arrest any energy drain. However, it is clear that the provision of nutrients per se will not replete losses of lean body mass or endogenous fat stores. The reason for this situation lies in the pathophysiological events that dictate the inflammatory response.

Returning to the energy balance equation, and more precisely energy input, an appreciation of the central role that inflammation has in relation to ingestive behaviour is not widely acknowledged. Recognition of the mechanisms that regulate or bring about dysfunction of such feeding behaviour in both acute and chronic disease may permit the development of strategies that would modulate the impact of the inflammatory response on dietary intake.

The inflammatory response initiated by the host can have profound effects on neurophysiological mechanisms responsible for ingestive behaviour (Konsman \& Dantzer, 2001). This process is primarily driven by the production of 
cytokines that orchestrate the host response to tissue injury, infection or inflammation (such as in pancreatitis and inflammatory bowel disease). Cytokines play a role in the anorexia of disease and in the altered energy metabolism and mobilisation of body mass and subsequent changes in body composition. For example, interleukin $1 \beta$ has been shown to have both direct central and indirect peripheral depressing effects on appetite mechanisms (Maier et al. 1998), whereas plasma concentrations of tumour necrosis factor $\alpha$ have been associated with increases in energy expenditure (Roubenoff et al.2002) and tissue wasting. In summary, the host response in terms of the presence of either disease or trauma induces loss of lean body mass and increased energy expenditure, which would contribute to disease-related undernutrition.

\section{Impact of cytokines on ingestive behaviour}

The anorectic effects of cytokines are likely to manifest as a disruption of the normal feeding behaviour. In a healthy individual feeding is regulated by neural and humoral signalling derived from gastrointestinal sensory nerves and by central control of appetite. In disease states the production of neurally-active cytokines will alter hypothalamic regulation of appetite because of both gut-derived afferent (sensory) sensitisation and centrally-mediated depression in motivation to eat (Fig. 1).

The ingestion of food elicits a number of reflexes that ultimately determine the quantity and quality of energy consumed. Oral ingestion of food acts as a potent positive stimulus for intake, providing of course that the food is palatable. However, the processes of satiation (termination of the meal) and satiety (continued inhibition of eating), which regulate the length of an eating episode and dictate the initiation of the next meal, are also partly dependent on oral sensation (French \& Cecil, 2001). Such inhibition of food intake is mediated by negative influences from the gut following ingestion, including gastric and intestinal mechano-receptor and chemo-receptor activation that is enhanced by incretin release (e.g. cholecystokinin). Following absorption, monitoring of the energy substrates and the available fuel mix in addition to those substrates being oxidised for energy have a role to play in the postabsorptive satiety mechanisms.
In the presence of inflammation a disturbance in this complex interplay of sensory, post-ingestive and postabsorptive mechanisms occurs that induces alterations in eating behaviour (Fig. 2). For example, sensory alterations in the chorda tympani (one of the neural pathways involved in the transduction of taste; Yanagisawa et al. 1998) induce changes in taste that may induce specific food preferences and may be a direct consequence of sensitisation by systemic inflammatory mediators (Phillips \& Hill, 1997). Such changes have been reported in neoplastic (De Wys \& Walters, 1975), liver (Madden et al. 1997) and inflammatory bowel diseases. Clinicians are unlikely to succeed in encouraging patients to consume an adequate oral intake. Indeed, studies from our group (Davidson et al. 1998) have shown that

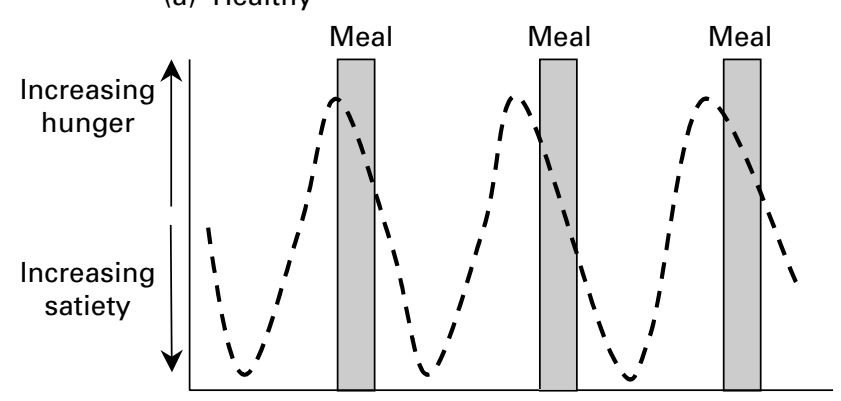

(b) Inflammatory conditions

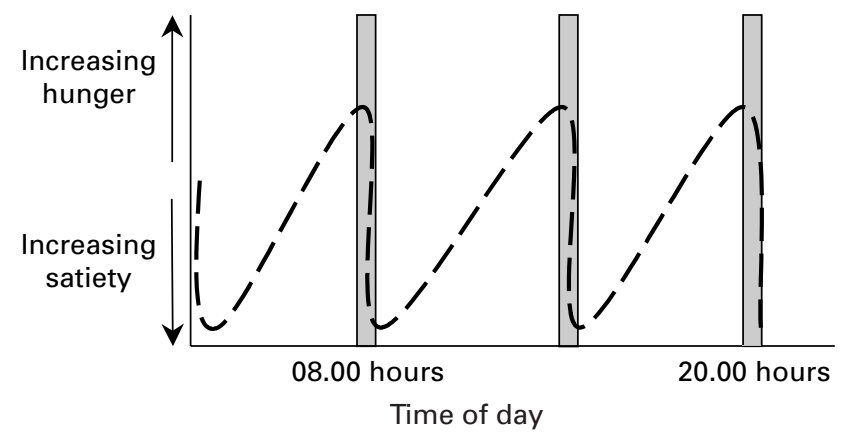

Fig. 2. Schematic representation of diurnal changes in hunger and satiety and their relationship with meal length in (a) normal and (b) inflammatory conditions.

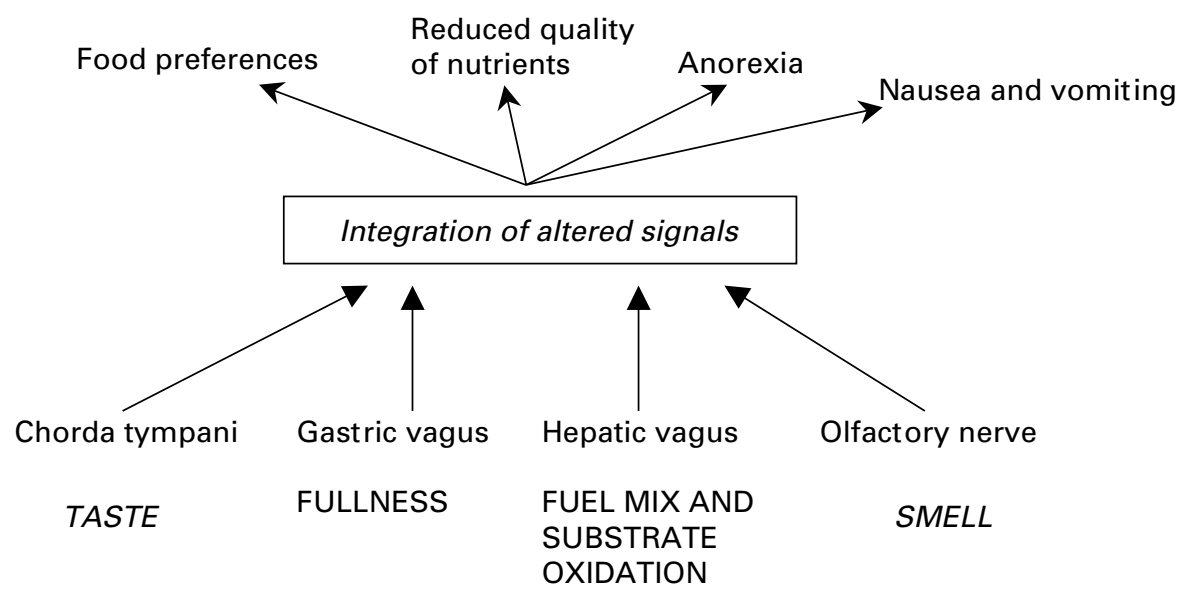

Fig. 1. Dietary consequences of alterations in gastrointestinal sensory signalling. 
patients with end-stage cancer have altered taste thresholds with respect to the bitter modality, and these changes are most apparent in those patients that are weight losing. Interestingly, patients with low bitter thresholds had an ongoing metabolic response, indicated by higher concentrations of C-reactive protein, interleukins $1 \beta$ and 6 and tumour necrosis factor $\alpha$. This work reveals an association between weight loss and the catabolic state in cancer patients and implicates immune cell-derived products in peripheral modulation of the gustatory system. Additionally, odour discrimination was also tested in this population, and the cancer population recognised significantly more than their age-matched counterparts. This exam-ple of altered gustatory and olfactory sensation illustrates the profound influence that mediators of the inflammatory response can have on dietary intake.

Analogous to this effect on food preference, sensitisation of the gastric vagus may result in increased activation of mechanisms mediating sensations of fullness that also contribute to the process of satiety. The outcome could be a lower satiation or satiety threshold in patients with ongoing inflammation, which is evidenced by the early satiety frequently reported by clinicians in practice. However, quantitative assessment of the impact of these effects in relation to dietary intake is rarely, if ever, undertaken in clinical practice. The presence of early satiety ensures a reduction in meal length and a poor intake. This response explains the poor dietary intakes in chronic inflammatory conditions such as Crohn's disease. However, what is less clear is whether this mechanism is peripheral (i.e. gut) and/or central (i.e. hypothalamic).

Subjective appetite variables have been assessed in patients with active Crohn's disease (Bannerman et al. 2001), showing differences in appetite ratings both at baseline (significantly lower hunger; $P<0 \cdot 05$ ) and following ingestion (significantly lower desire to eat $P<0 \cdot 05$ ). This finding suggests that dietary intake is limited by motivation to eat and by enhanced appetite responses that promote inhibition of eating following ingestion. It should be noted that in the work of Bannerman et al. (2001) water was used as the ingestive stimulus and not food (nutrients), which may well have underestimated the changes in subjective appetite variables that actually occur in this group. This underestimation is primarily because in this experimental situation the incretin-mediated mechanisms involved in the satiety cascade after food is ingested would not have been initiated.

Acute and chronic disease may place constraints on normal feeding behaviour, and it is not surprising that marked alterations in metabolic profiles have been reported. In Crohn's disease the response includes an enhanced fasting profile (Rigaud et al. 1994) with increased reliance on fat oxidation for energy production, and in other inflammatory conditions (trauma or surgical intervention) an extent of insulin resistance occurs. In the presence of such metabolic abnormalities it would be appropriate to expect an increase in motivation to eat in order to arrest the enhanced fasting condition and the reduced availability of the preferred fuel at the cellular level. However, this response is clearly not present, which is probably because the blood glucose profiles mirror those occurring in the (late) postprandial state and the post-absorptive satiety mechanisms facilitated by the liver may be stimulated because of increased hepatic energy demands. The role of the liver in ingestive behaviour is one of an energy sensor (Friedman, 1997), and during inflammation the production of acute-phase protein, an energy-demanding process, is up regulated. Consequently, ATP-ADP turnover will be increased and the absolute energy status of the liver may not change. This process explains why the hyperphagic stimulus that occurs with the reduction in available energy in the liver (Horn et al. 1999) does not occur in acute and chronic illness.

As a result of the changes in appetite regulatory mechanisms seen in disease the provision of energy and macro- and micronutrients to undernourished patients with inflammation is unlikely to achieve anabolism. However, an appreciation of strategies that attenuate the inflammatory response may provide an opportunity to re-establish energy homeostasis and replete lean body mass. It should be remembered that for patients and their carers weight loss has been cited as one of the most troublesome symptoms of their disease (Curtis et al. 1991; Holden, 1991).

\section{Strategies to attenuate the consequences of inflammation}

Whilst a reduction in energy intake seems almost a ubiquitous problem in acute and chronic disease, there is a paucity of literature that has examined spontaneous intake. Kondrup \& Muller (1997) observed that the oral intake of patients with liver disease was only 50 and $57 \%$ of the requirements for energy and protein respectively. Similarly, in one of the few studies that considered oral intake in cancer patients, Holmes \& Dickerson (1991) found that energy and protein intakes were 55 and $78 \%$ of the requirements respectively. Attempts have been made to increase dietary intake using pharmacological agents such as anabolic steroids, megestrol acetate, that stimulate appetite. The results have proved disappointing (Yeh et al. 2000), with any effect on appetite being transient in nature and, as might be expected, any weight gain was fat and not lean tissue (Burrowes et al. 1999).

Another avenue of investigation is the oral administration of eicosapentaenoic acid, which down regulates the acutephase protein response, in an attempt to attenuate the weight loss and anorexia of inflammatory disease (Calder \& Grimble, 2002). For example, Wigmore et al. (1996) supplemented patients with irresectable pancreatic cancer with fish oil $(12 \mathrm{~g} / \mathrm{d})$. Before supplementation patients had a median weight loss of $2.9 \mathrm{~kg} / \mathrm{month}$ and after 12 weeks on fish oil had a weight gain of $0.3 \mathrm{~kg} /$ month. Importantly, this weight gain could be attributed to some improvement in lean body mass. Work by the same group (Barber et al. 1999) showed that provision of an energy-dense oral supplement enriched with eicosapentaenoic acid $(1 \cdot 1 \mathrm{~g} / \mathrm{d})$ over 8 weeks increased appetite and lean body mass, and reduced energy expenditure in patients with advanced cancer. Similarly, a number of studies have examined the use of fish oil to dampen the effects of the inflammation in order to reduce disease activity and severity (Simopoulos, 2002). However, the impact of this treatment modality on nutritional status in conditions other than pancreatic cancer remains to be elucidated. 


\section{Energy requirements}

Whilst the present paper has focused on the influence of the inflammatory response on energy balance, another innovative nutritional strategy that may be important in attenuating the metabolic response to major open surgery is pre-operative carbohydrate loading. Typically, patients can be fasted for $\leq 16 \mathrm{~h}$ before surgery, at which time liver glycogen stores will be virtually exhausted. This fast combined with the surgical insult may detrimentally impact on clinical outcome. The provision of pre-operative carbohydrate has been shown to depress the post-operative metabolic response, improve insulin resistance and reduce recovery time (Nygren et al. 1998; Soop et al. 2000). Recently, a double-blind randomised study (placebo $v$. carbohydrate fluids) conducted by our group has shown that carbohydrate provision before major surgery significantly preserves muscle mass $(P<0 \cdot 05$; Yuill et al. 2002). However, the question remaining is whether this preservation of muscle mass is reflected in improved functional performance, thus warranting further investigation.

In acute and chronic disease the determination of energy requirements is pivotal in sustaining energy balance. Predictive equations have now been developed for criticallyill populations (Ireton-Jones et al. 1992), although accurate prescribing of energy intake in the acute setting is uncommon. Only 25-32\% of the patients have energy intakes within $10 \%$ of their requirements (McClave et al. 1997). Clearly, the variation in energy expenditure has led to difficulties in accurately defining the energy needs of the individual. This situation is of concern because it may lead to under- or overfeeding and further compromise the patient's clinical condition. It appears that practitioners must be cognisant of the limitations of calculating energy requirements.

In conclusion, therefore, it would appear that there are two approaches that may be adopted in order to ensure that the nutritional demands of acute and chronic illness are met. The first approach is a thorough appreciation of the diseaseinduced alterations in hunger and satiety and the identification of the optimal timing for nutritional intake or supplementation (Wilson et al. 2002). The second approach is the attenuation of the inflammatory response by the pre-operative management of major elective surgery or by the amelioration of the ongoing inflammatory conditions associated with acute and chronic disease.

\section{References}

Bannerman E, Davidson I, Conway C, Culley D, Aldhous M \& Ghosh S (2001) Altered subjective appetite parameters in Crohn's disease. Clinical Nutrition 20, 399-405.

Barber MD, Ross JA, Voss AC, Tisdale MJ \& Fearon KC (1999) The effect of an oral supplement enriched with fish oil on weightloss in patients with pancreatic cancer. British Journal of Cancer 81, 80-86.

Burrowes JD, Bluestone PA, Wang J \& Pierson RN (1999) The effects of moderate doses of megestrol acetate on nutritional status and body composition in a hemodialysis patient. Journal of Renal Nutrition 9, 89-94.

Calder PC \& Grimble RF (2002) Polyunsaturated fatty acids, inflammation and immunity. European Journal of Clinical Nutrition 56, Suppl. 3, S14-S19.
Curtis E, Krech R \& Declan-Walsh T (1991) Common symptoms in patients with advanced cancer. Journal of Palliative Care 7 , 25-29.

Davidson HIM, Pattison RM \& Richardson RA (1998) Clinical undernutrition states and their influence on taste. Proceedings of the Nutrition Society 57, 633-638.

Davidson HIM, Richardson RA, Sutherland D \& Garden OJ (1999) Macronutrient preference, dietary intake and substrate oxidation among stable cirrhotic patients. Hepatology 29, 1380-1386.

DeWys WD \& Walters K (1975) Abnormalities of taste sensation in cancer patients. Cancer 36, 1888-1896.

French SJ \& Cecil JE (2001) Oral, gastric and intestinal influences on human feeding. Physiology and Behavior 74, 729-734.

Friedman MI (1997) An energy sensor for control of energy intake. Proceedings of the Nutrition Society 56, 41-50.

Holden CM (1991) Anorexia in the terminally ill cancer patient: the emotional impact on patient and the family. Hospital Journal 7, 73-84.

Holmes S \& Dickerson WT (1991) Food intake and quality of life in cancer patients. Journal of Nutritional Medicine 2, $359-368$.

Horn CC, Addis A \& Friedman MI (1999) Neural substrate for an integrated metabolic control of feeding behavior. American Journal of Physiology 276, R113-R119.

Ireton-Jones CS, Turner WW, Leipa GU \& Baxter C (1992) Equations or the estimation of energy expenditures in patients with burns with special references to ventilatory status. Journal of Burn Care and Rehabilitation 13, 330-333.

Kondrup J \& Müller MJ (1997) Energy and protein requirements of patients with liver disease. Journal of Hepatology 27, 239-247.

Konsman JP \& Dantzer T (2001) How the immune and nervous systems interact during disease-associated anorexia. Nutrition 17, 664-668.

McClave SA, Sinder H, Owens N \& Sexton L (1997) Clinical nutrition in pancreatitis. Digestive Diseases and Science 42, 2035-2044.

Madden A, Bradbury W \& Morgan M (1997) Taste perception in cirrhosis: its relationship to circulating micronutrients and food preferences. Hepatology 26, 40-48.

Maier SF, Goehler LE, Fleshner M \& Watkins LR (1998) The role of the vagus nerve in cytokine to brain communication. Annals of the New York Academy of Sciences 840, 289-300.

Nygren J, Soop M, Thorell A, Efendic S, Nair KS \& Ljungqvist O (1998) Preoperative oral carbohydrate administration reduces postoperative insulin resistance. Clinical Nutrition 17, $65-71$.

Phillips LM \& Hill DL (1997) Novel regulation of peripheral gustatory function by the immune system. American Journal of Physiology 271, R857-R862.

Rigaud D, Angel LA, Cerf M, Carduner MJ, Melchior JC, Sautier C, Rene E, Apfelbaum M \& Mignon M (1994) Mechanisms of decreased food intake during weight loss in adult Crohn's disease patients without obvious malabsorption. American Journal of Clinical Nutrition 60, 775-781.

Roubenoff R, Grinspoon S, Skolink PR, Tchetgen E, Abad L, Spiegelman P, Knox T \& Gorbach S (2002) Role of cytokines and testosterone in regulating lean body mass and resting energy expenditure in HIV-infected men. American Journal of Physiology 283, E138-E145.

Schols AMWJ (2001) Nutrition and respiratory disease. Clinical Nutrition 20, Suppl. 1, 173-179.

Simopolous AP (2002) Omega-3 fatty acids in inflammation and autoimmune diseases. Journal of the American College of Nutrition 21, 495-505. 
Soop M, Nygren J, Myrefors P, Thorell A \& Ljungqvist (2000) Preoperative oral carbohydrate treatment attenuates immediate postoperative insulin resistance. American Journal of Physiology 280, E567-E583.

Wigmore S, Ross J, Falconer JS, Plester CE, Tisdale MJ, Carter DC $\&$ Fearon KC (1996) The effect of polyunsaturated fatty acids on the progress of cachexia in patients with pancreatic cancer. Nutrition 12, 27-30.

Wilson MM, Purushothaman R \& Morely JE (2002) Effect of liquid dietary supplement on energy intake in the elderly. American Journal of Clinical Nutrition 75, 944-947.
Yanagisawa K, Bartoshuk LM, Catalanotto FA, Karrer TA \& Kveton JF (1998) Anaesthesia of the chorda tympani nerve and taste phantoms. Physiology and Behavior 63, 29-35.

Yeh SS, Wu SY, Lee TP, Olson JS, Stevens MR, Dixon T, Porcelli RJ \& Schuster MW (2000) Improvement in quality-of-life measures and stimulation of weight gain after treatment with megestrol acetate oral suspension in geriatric cachexia: results of a double-blind, placebo-controlled study. Journal of the American Geriatrics Society 48, 485-492.

Yuill KA, Parks R, Richardson RA, Davidson HIM \& Garden OJ (2002) Oral administration of carbohydrate-containing fluids prior to major elective surgery preserves lean tissue postoperatively. British Journal of Surgery 89, Suppl. 1, 92. 